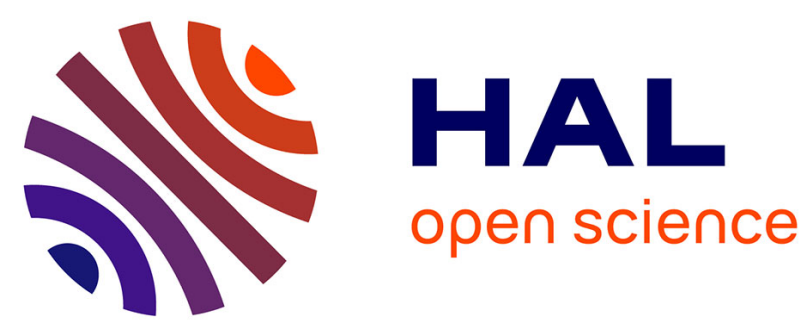

\title{
High energy synchrotron radiation. A new probe for condensed matter research
}

Jodi Schneider, R. Bouchard, T. Brückel, M. Lippert, H.-B. Neumann, H.

Poulsen, U. Rütt, T. Schmidt, M. von Zirnmermann

\section{To cite this version:}

Jodi Schneider, R. Bouchard, T. Brückel, M. Lippert, H.-B. Neumann, et al.. High energy synchrotron radiation. A new probe for condensed matter research. Journal de Physique IV Proceedings, 1994, 04 (C9), pp.C9-415-C9-421. 10.1051/jp4:1994969 . jpa-00253532

\section{HAL Id: jpa-00253532 https://hal.science/jpa-00253532}

Submitted on 1 Jan 1994

HAL is a multi-disciplinary open access archive for the deposit and dissemination of scientific research documents, whether they are published or not. The documents may come from teaching and research institutions in France or abroad, or from public or private research centers.
L'archive ouverte pluridisciplinaire HAL, est destinée au dépôt et à la diffusion de documents scientifiques de niveau recherche, publiés ou non, émanant des établissements d'enseignement et de recherche français ou étrangers, des laboratoires publics ou privés. 


\section{High energy synchrotron radiation. A new probe for condensed matter research}

J.R. Schneider, R. Bouchard, T. Brückel, M. Lippert, H.-B. Neumann, H.F. Poulsen, U. Rütt,

T. Schmidt and M. von Zimmermann

Hamburger Synchrotronstrahlungslabor HASYLAB at Deutsches Elektronen-Synchrotron DESY, Notkestr. 85, 22603 Hamburg, Germany

The absorption of $150 \mathrm{keV}$ synchrotron radiation in matter is weak and, as normally done with neutrons, bulk properties are studied in large samples. However, the $\mathbf{k}$-space resolution obtained with a Triple Crystal Diffractometer (TCD) for high energy synchrotron radiation is about one order of magnitude better than in high resolution neutron diffraction.

The technique has been applied to measure the structure factor $S(Q)$ of amorphous solids up to momentum transfers of the order of $32 \AA^{-1}$, to study the intermediate range Ortho-II ordering in large, high quality $\mathrm{YBa}_{2} \mathrm{Cu}_{3} \mathrm{O}_{6.5}$ single crystals and for investigations of the defect scattering from annealed Czochralski grown silicon crystals. Magnetic superlattice reflections have been measured in $\mathrm{MnF}_{2}$ demonstrating the potential of the technique for high resolution studies of ground state bulk antiferromagnetism. Recently the question of two length scales in the critical scattering at the $100 \mathrm{~K}$ phase transition in $\mathrm{SrTiO}_{3}$ was studied.

At the PETRA storage ring, which serves as an accumulator for the HERA electron-proton-ring at DESY and which can be operated up to electron energies of $12 \mathrm{GeV}$, an undulator beam line is currently under construction and should be available in summer 1995. It opens up exciting new research opportunities for photon energies from about 20 to $150 \mathrm{keV}$.

\section{INTRODUCTION}

Modern insertion devices at present day storage rings operated with electrons or positrons with energies above $4 \mathrm{GeV}$ provide intense X-ray beams with wavelengths of the order of $0.1 \AA$. In the near future the accessible wavelength range will be extended down to $0.01 \AA$. The use of such short wavelength synchrotron radiation opens up interesting new fields in diffraction physics, both for inelastic and elastic scattering experiments. Photons with energies of the order of $150 \mathrm{keV}$ are only weakly absorbed in matter. For example, the mean free path is about $3 \mathrm{~cm}$ for $\mathrm{Si}$ and $0.6 \mathrm{~cm}$ for Fe. Bragg angles are of the order of a few degrees (Fig. 1) and therefore the polarization factor is close to unity. In most cases the scattering from imperfect single crystals can be interpreted within first order Born approximation, or only small corrections to it are needed. Therefore the diffraction of high energy photons has much in common with neutron diffraction and the same samples can be used in both types of experiments. In the past, 
most experiments with short wavelength photons have been performed with $\gamma$-radiation from radioactive sources, a typical wavelength being $0.04 \AA$ [1-4].

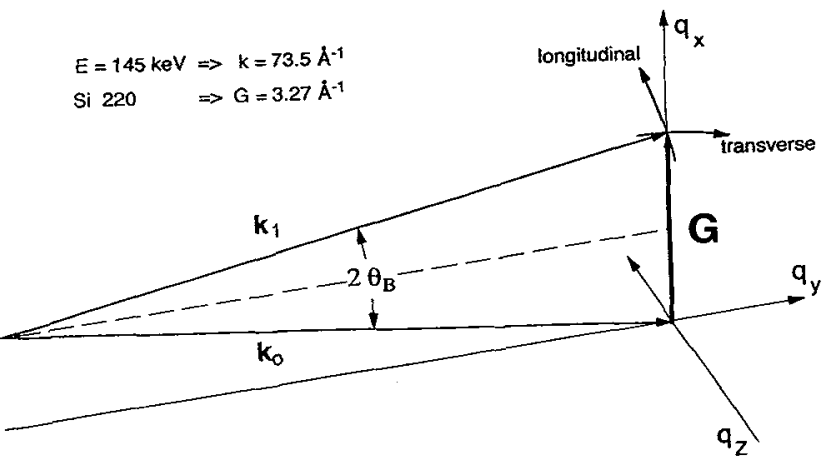

Fig. 1: Scattering geometry in wave vector space for the diffraction of $145 \mathrm{keV}$ synchrotron radiation at silicon 220. $G$ represents the reciprocal lattice vector.
The first triple crystal diffractometer (TCD) mesasurements with high energy synchrotron radiation have been performed at CHESS in Cornell, USA [5]. The main components of such a TCD are an absorber, which suppresses the radiation with energies smaller than $\sim 40$ $\mathrm{keV}$, a monitor counter, a monochromator, the sample, an analyzer, and a solid state detector. By appropriate rotations of the sample and analyzer crystals the region around reciprocal lattice points is scanned. Because of the small Bragg angles the samples are studied in transmission (Laue) geometry.

In the present paper, after a discussion of the $k$-space resolution of a TCD, we report on experiments with short wavelength synchrotron radiation performed recently at HASYLAB with a new diffractometer with its scattering plane in the horizontal allowing for easy mounting of large cryostats and furnaces.

\section{RESOLUTION OF THE TRIPLE CRYSTAL DIFFRACTOMETER}

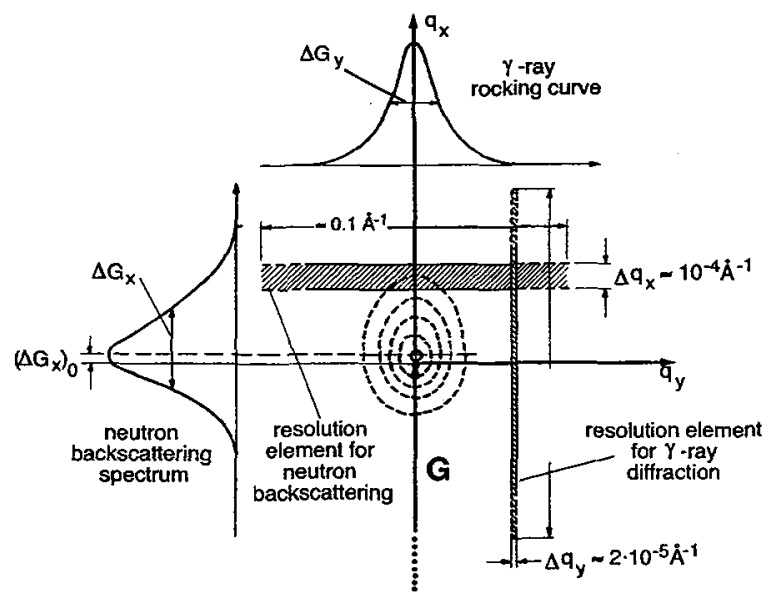

Fig. 2: Scheme for the characterization of crystal perfection in reciprocal space: the dashed contour lines indicate the distribution of Bragg scattered intensity with its center displaced by $\left(\Delta G_{x}\right)_{0}$ with respect to the position of the reciprocal lattice point $\boldsymbol{G}$ in an ideal lattice, due to a change of the mean value of the lattice parameter $d . \Delta G_{x} / G=\Delta d / d$ describes the lattice parameter fluctuations caused by strain. The so called mosaic-spread $\Delta G_{y} / G$ is a measure for the lattice plane tilts introduced by the defects.
The main features of the resolution function of a triple crystal diffractometer for high energy synchrotron radiation may be best introduced by comparing it with the $\mathbf{k}$-space resolution of a high resolution neutron backscattering spectrometer [6] and a double crystal $\gamma$ ray diffractometer [7] with respect to the mutual possibilities for the characterization of the degree of perfection of imperfect crystals, i.e. by mapping out the scattering power around a reciprocal lattice point which strongly depends on strain and lattice plane tilts in the sample [8]. The relevant quantities are defined in Fig. 2.

Backscattering diffractometers integrate in $\mathbf{k}$-space over planes perpendicular to $\mathbf{G}$ and no information on lattice plane tilts is obtained. The thickness of the sheet of integration is of the order of $10^{-4} \AA^{-1}$, however, the lattice plane 
spacings of monochromator and analyzer must not differ by more than $\Delta \mathrm{d} / \mathrm{d} \sim 10^{-4}$. In $\gamma$-ray diffractometry the resolution element is a disk in $\mathbf{k}$-space parallel to $\mathbf{G}$ approximately $2 \cdot 10^{-5} \AA^{-1}$ thick. Therefore crystal mosaicity can be studied with high resolution but no information on changes in lattice parameters is obtained.
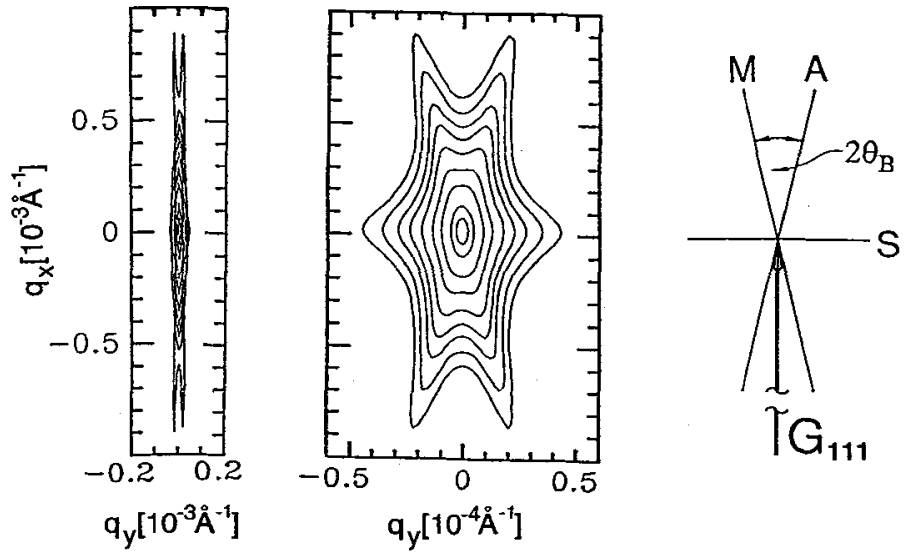

A TCD for high energy synchrotron radiation combines the good longitudinal resolution of neutron backscattering with the excellent lateral resolution of a double crystal $\gamma$-ray diffractomter and allows for a fine mesh scanning of $\mathbf{k}$-space. Perfect crystals, used as monochromator and analyzer, have a Lorentzian shaped diffraction pattern. Therefore the resolution function of a $\mathrm{TCD}$ shows tails in $\mathbf{k}$-space and their

Fig. 3: Intensity distribution of a TCD calculated for $80 \mathrm{keV}$ synchrotron radiation diffracted from (111) lattice planes of 3 perfect crystals in non-dispersive Laue geometry.

location has to be known very well [9]. As shown in Fig.3 for the dispersion free setting the resolution tails due to the diffraction patterns of monochromator (M), sample (S) and analyzer (A) are confined to a very narrow stripe parallel $\mathbf{G}$, mainly because of the small Bragg angles at high photon energies. In addition the FWHM of the diffraction pattern is proportional to the wavelength $\lambda$, the intensity in the wings of the Lorentzian even decays with $\lambda^{2}$. In dispersive settings the resolution function is more complicated but again it is well understood [10].

The following applications of TCD with high energy synchrotron radiation to problems in condensed matter physics have been selected in order to emphasize the complementarity of the technique to neutron scattering.

\section{STRUCTURE FACTORS OF AMORPHOUS MATERIALS}

The use of hard X-rays (60-300 keV) for diffraction studies of disordered materials has several advanta-

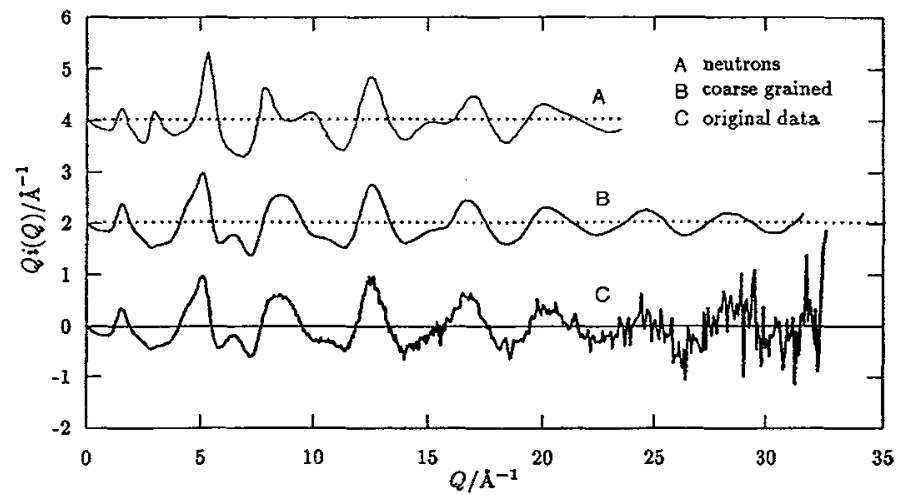

Fig. 4: First moment of the interference function of silica as a function of momentum transfer $Q$ measured with $95 \mathrm{keV}$ photons [11] and neutrons [13], respectively. ges: higher resolution in direct space, smaller corrections for absorption and multiple scattering, removal of truncation effects, the possibility for operating in extreme environments and the direct comparison between X-ray and neutron data. A feasibility study for amorphous silica has been performed recently with $95 \mathrm{keV}$ radiation from a HASY$\mathrm{LAB}$ wiggler beam line and a cylindrical sample of $3 \mathrm{~mm}$ diameter [11]. The resulting systematic errors intrinsic to the scattering process (not including errors in the form 
factors) are found to be of the order of $0.2 \%$. Fig. 3 shows the first moment of the interference function as defined by Warren [12] together with a neutron diffraction result [13].

\section{SINGLE CRYSTAL STRUCTURE DETERMINATION}

Charge density studies with $\gamma$-ray diffractometry [4] have demonstrated the power of hard X-rays in accurate structure work. The main advantages of this approach are due to the weak absorption and small

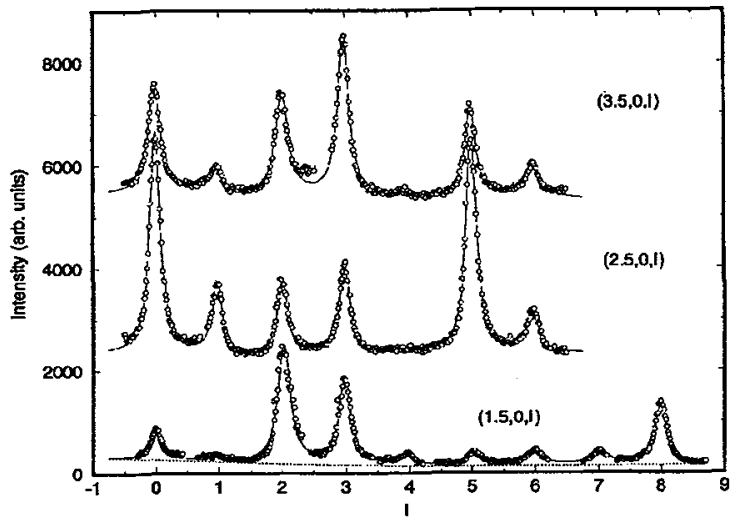

Fig. 5: Plot of the Ortho-II superstructure reflections along $[1.5,0,1],[2.5,0,1]$ and $[3.5,0,1]$. The solid lines are the fitted Lorentzians. The dashed line for the bottom scan shows the fitted background. extinction, anomalous dispersion can be neglected and direct comparison with neutron data taken at the same sample is possible. Unfortunately the intensities are weak and very long measuring times are required. In order to improve on this a 4-circle diffractomter for high energy synchrotron radiation has been set up at a HASYLAB wiggler beamline recently and was applied to investigations of the equilibrium bulk structural properties of a $\mathrm{YBa}_{2} \mathrm{Cu}_{3} \mathrm{O}_{6.5}$ single crystal, $3.5 \cdot 3.5 \cdot 0.65 \mathrm{~mm}^{3}$ in dimension [14]. The crystal from the Phys. Dep. of the Univ. of British Columbia, Canada, was found to display record braking FWHM of the superstructure peaks: $\Delta \mathrm{h}=0.024, \Delta \mathrm{k}<0.01$ and $\Delta \mathrm{l}=0.14$ (dimensionless units).

The atomic displacement parameters obtained with $101.3 \mathrm{keV}$ synchrotron radiation are in good agreement with earlier X-ray measurements, however, a detailed analysis of these new results in combination with the neutron data taken at Ris $\emptyset$ Nat. Laboratory, Denmark, on the same sample is still under way.

\section{DIFFUSE SCATTERING FROM OXYGEN INDUCED DEFECTS IN SILICON}

Czochralski grown silicon crystals widely used in semiconductor industry contain about $10 \mathrm{ppm}$ oxygen atoms. When annealed at temperatures around $750^{\circ} \mathrm{C}$ large $\mathrm{SiO}_{2}$ precipitates are formed, their shape and number density is well determined by small angle neutron scattering (SANS)[15]. At temperatures above

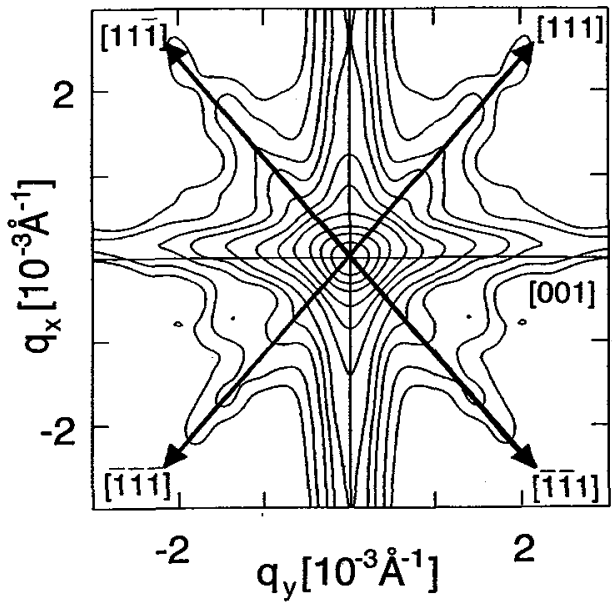

$900^{\circ} \mathrm{C}$ strain relaxes by forming secondary defects like stacking faults and dislocation loops. They produce the characteristic pattern of diffuse scattering shown in Fig.6. The contribution of the two types of defects to the diffuse scattering becomes clearer in the individual longitudinal scans, one of which is shown in Fig.7. In contrast to the dislocation loops (Fig.8) the FWHM of the streaks due to stacking faults is independent of $q$.

Fig. 6: Measured diffuse scattering in the vicinity of reflection 220 of a silicon crystal annealed for $24 \mathrm{~h}$ at $750^{\circ} \mathrm{C}$ and $20 \mathrm{~h}$ at $1050^{\circ} \mathrm{C}$. The streaks along $\langle 111\rangle$ are due to stacking faults on [111\} planes with Burgers vector $\langle 111\rangle$, the streaks along $\langle 001\rangle$ are due to dislocation loops on $\{111\}$ with Burgers vector $\langle 110\rangle$. 


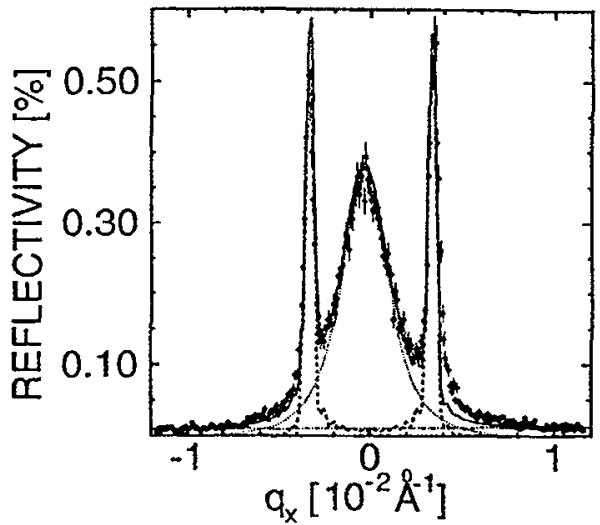

Fig. 7: Longitudinal scan at $q_{y}=2.4 \cdot 10^{-3} \AA^{-1}$ through the diffuse scattering shown in Fig.6. The two sharp peaks are due to the stacking faults, the wider central peak is due to the dislocation loops.

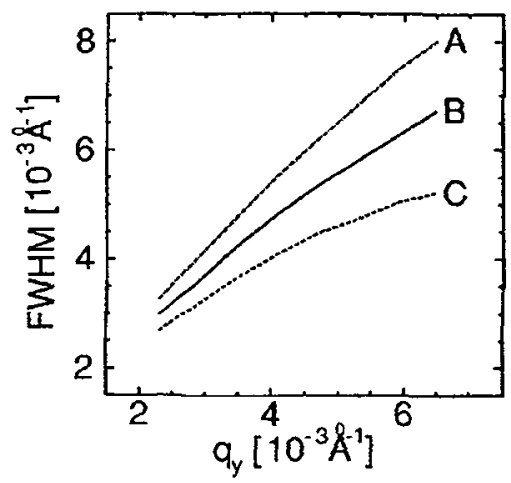

Fig. 8: q[001]-dependence of the FWHM of the central peak in Fig.7 calculated according to Larson\&Schmatz [16] for dislocation loops with a radius of $100(\mathrm{~A}), 250(\mathrm{~B})$ and $500 \AA(\mathrm{C})$.

At present about $15 \mathrm{~min}$. are needed in order to measure a scan of the type shown in Fig.7. This allowed in-situ studies of the annealing processes [17]. They will become much more attractive in the near future, when radiation from the undulator beam line under construction at the PETRA storage ring at DESY will be available and where such scans can be performed much faster. Then the formation of the secondary defects can be studied in-situ for annealing cycles applied during device production.

\section{INVESTIGATION OF GROUND STATE ANTIFERROMAGNETISM}

Today, the most powerful methods for the investigation of magnetic structures are magnetic neutron scattering and synchrotron-X-ray scattering in the energy range 3-15 keV. The use of high energy syn-

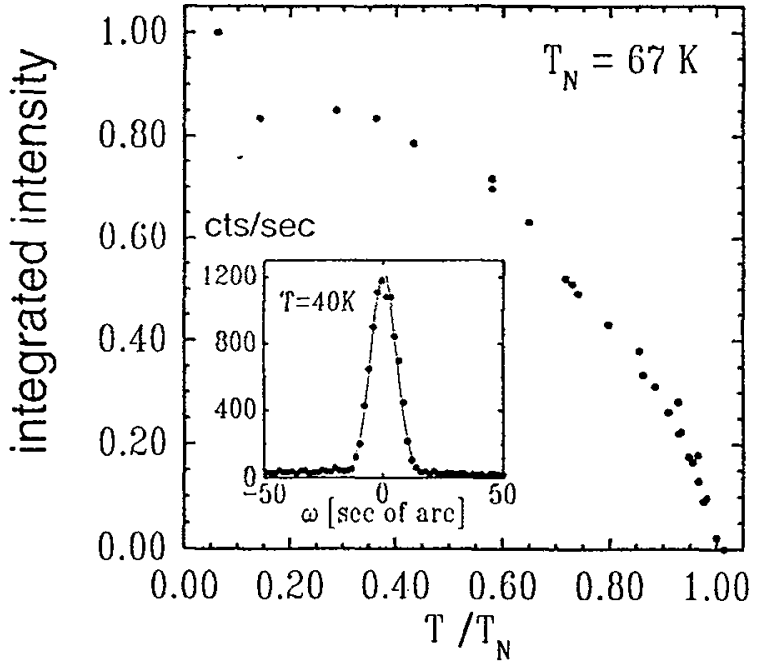

Fig. 9: Temperature dependence of the integrated reflecting power of the magnetic $\mathrm{MnF}_{2} 300$ reflection measured with $80 \mathrm{keV}$ synchrotron radiation. The insert shows a transverse scan with FWHM $=12.3^{\prime \prime}$, the FWHM of the longitudinal scan is 6.3". chrotron radiation combines some of the advantages of the afore mentioned techniques: high $\mathbf{k}$-space resolution and bulk sensitivity. In addition, the purely magnetic cross section reduces to a good approximation to the simple formula

$$
(d \sigma / d \Omega)_{\text {magneric }}=\left(\lambda_{c}^{2} / d^{2}\right) \cdot\left|S_{2}^{2}\right|
$$

$\lambda_{c}$ is the Compton wavelength and $d$ the lattice parameter. $S_{2}$ is the component of the Fourier transform of the spin density perpendicular to the scattering plane.

Fig.9 shows the results obtained with a TCD using $80 \mathrm{keV}$ radiation from a HASYLAB wiggler beam line and annealed silicon crystals as monochromator and analyzer, respectively [18]. The peak count rate of $1400 \mathrm{cps}$ is remarkable, the excellent $\mathbf{k}$-space resolution is obvious. 


\section{STUDIES OF STRUCTURAL PHASE TRANSITIONS}

High k-space resolution and extinction free measurements of Bragg peaks are, together with high intensities for the measurement of weak superlattice reflections and/or critical scattering, essential for studies of structural phase transitions by diffraction techniques. Using a TCD and high energy synchrotron radia-

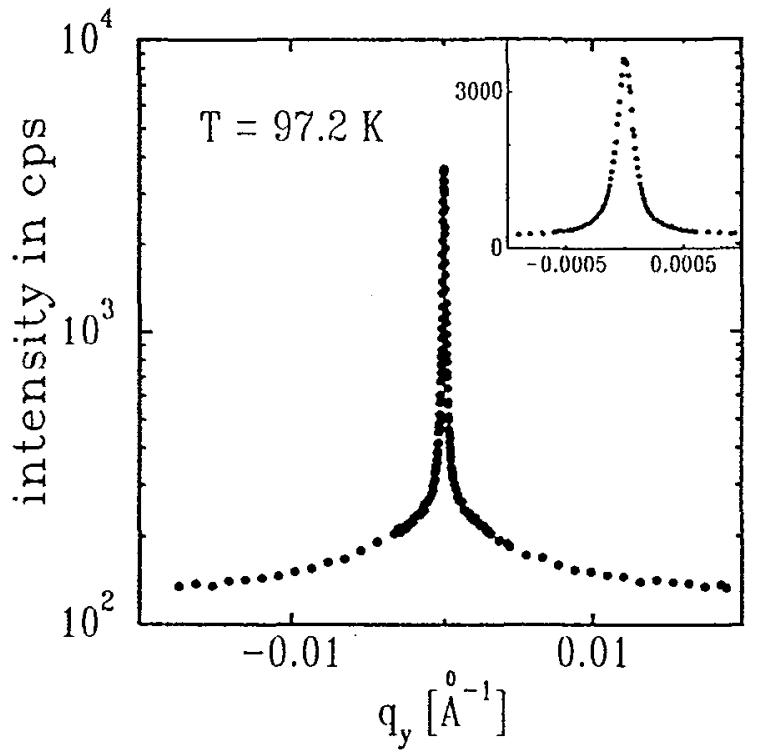

Fig. 10: Transverse scan $\left(q_{x}=0 ; 95 \mathrm{keV}\right)$ approximately $2 K$ below $T_{c}$ at reflection $1 / 2(511)$ on the identical $\mathrm{SrTiO}_{3}$ sample on which the original neutron work [20] has been done. The FWHM of the sharp component of $1.6 \cdot 10^{-4} \AA^{-1}$ is equal to the TCD resolution obtained with annealed Si crystals as monochromator and analyzer. tion not only bulk properties are probed, but the sample volume from which the photons are scattered can be confined to a narrow slice parallel to the surface if the considered reciprocal lattice vector is parallel to it. Thus changes in the scattering between the pure bulk and the surface near region can be studied.

In 1986, Andrews reported an unexpected twocomponent line shape observed from X-ray scattering measurements of the critical scattering associated with the structural phase transition in $\mathrm{SrTiO}_{3}$ [19]. At temperatures closer to $\mathrm{T}_{\mathrm{c}}$, the critical scattering was qualitatively changed by the appearance of a very narrow peak superimposed on the already present broad peak. This implies the existence of a second, and much larger, length scale just above $T_{c}$. This discovery is remarkable in view of the current understanding of critical phenomena, in which only one correlation length is needed to characterize the average size of the fluctuating regions above $\mathrm{T}_{\mathrm{c}}$. There is discussion to what extent this surprising re-

sult is due to the fact that $\mathrm{X}$-rays probe the surface near region. Because TCD with high energy synchrotron radiation combines high $\mathbf{k}$-space resolution and bulk sensitivity this technique should be able to solve the problem.

First measurements have been done on the same sample on which the original neutron scattering work was performed at Brookhaven Nat. Lab. [20] and Fig.10 shows a transverse scan at a temperature approximately $2 \mathrm{~K}$ below $\mathrm{T}_{\mathrm{c}}$. The sharp peak in the center is due to Bragg scattering and demonstrates the obtained resolution. The critical scattering can be measured without problems.

\section{AN UNDULATOR BEAMLINE AT THE PETRA STORAGE RING}

Because of the weak interaction between hard X-rays and matter sources of high brightness are needed to make full use of the potential of high energy X-ray diffraction for condensed matter research. In 1993 DESY decided to build an undulator beamline at the PETRA storage ring, which is part of the HERA injection system and which ramps electrons from 7 to $12 \mathrm{GeV}$. For photon energies above $20 \mathrm{keV}$ the PETRA undulator will provide a beam of synchrotron radiation with worldwide unique characteristics. Technical details are given in the HASYLAB 1993 Annual Report [21]. The beam will be operated as a DESY test facility and can be used for synchrotron radiation experiments whenever PETRA is not needed for injection of electrons or protons into HERA or for machine studies. From the performance of HERA in 1993, the second year of its operation, one can conclude that at least $50 \%$ of the running time 
of HERA should be available for synchrotron radiation experiments with the PETRA undulator.

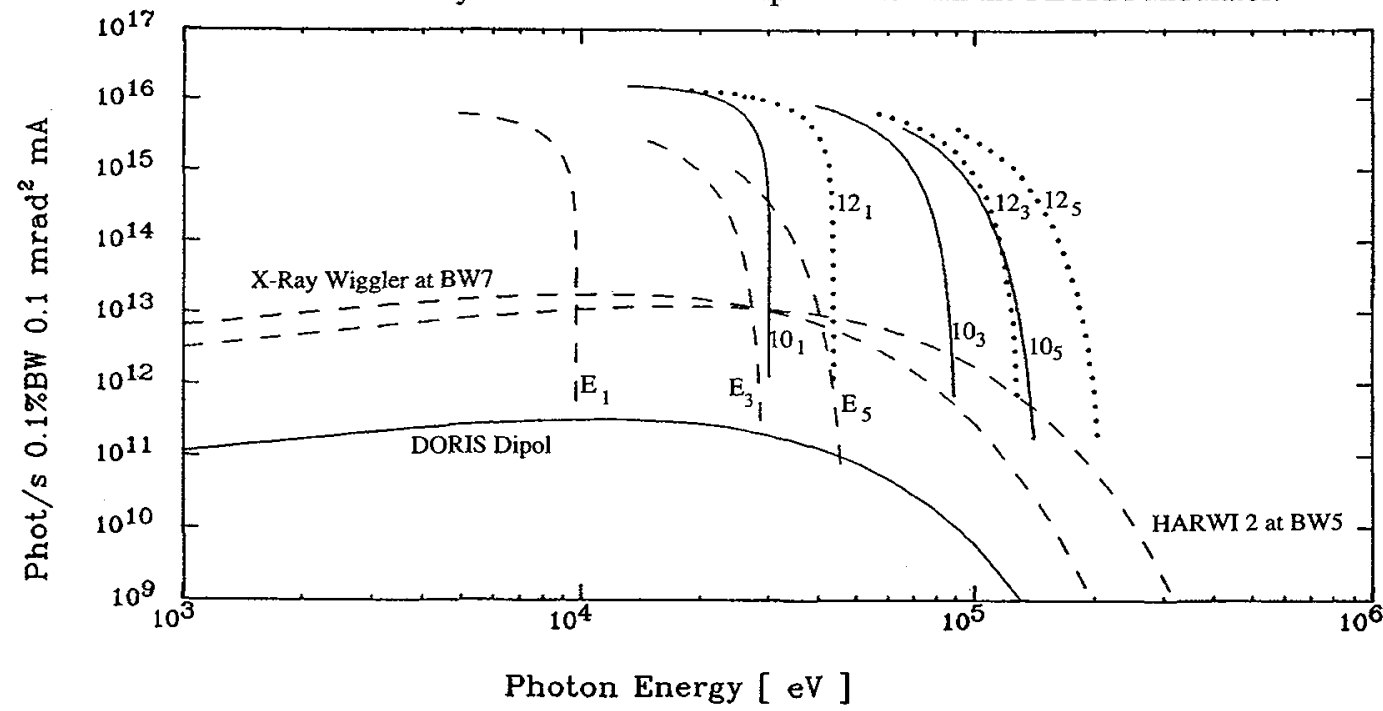

Fig. 11: Plot of the brightness of the 1st, 3rd and 5th harmonic of the PETRA undulator for electron energies of $10 \mathrm{GeV}$ (full) and $12 \mathrm{GeV}$ (dotted). For comparison the corresponding curves for an ESRF undulator $(E)$ as well as for the standard DORIS-III X-ray wiggler, the new high field DORIS wiggler (HARWI 2) and a DORIS dipole magnet at $5.3 \mathrm{GeV}$ are shown (parameters: J. Pflüger [21]).

\section{REFERENCES}

[1] Schneider J.R., Nuclear Science Applications, vol.2, (Harwood, UK, 1981) 227-276.

[2] Schneider J.R., Jørgensen J.E. and Shirane G., Phase Transitions8 (1986) 17-34.

[3] Bastie P., Troussaut F., Vallade M. and Zeyen C.M.E., J. Appl. Cryst.20 (1987) 475-481.

[4] Palmer A. and Jauch W., Phys. Rev. B 48 (1993) 10304-10310.

[5] Siddons D.P., Hastings J.B., Schneider J.R. and Berman L.E., Rev. Sci. Instr. 60 (1989) 2398-2401

[6] Birr M., Heidemann A. and Alefeld B., Nucl. Instrum. Methods 95 (1971) 435-440.

[7] Schneider J.R. and Graf H.A., J. Crystal Growth 74 (1986) 191-202.

[8] Bouchard R., Kouptsidis S., Neumann H.B., Schmidt T. and Schneider J.R., J. Appl. Phys.73 (1993) 3680-3684.

[9] Neumann H.B., Rütt U., Bouchard R. and Schneider J.R., J. Appl. Cryst., submitted

[10] Rütt U., Neumann H.B., Poulsen H.F. and Schneider J.R., J. Appl. Cryst., to be submitted

[11] Poulsen H.F., Neumann H.B., Schneider J.R., Neuefeind J. and Zeidler M.D., HASYLAB Annual Report (1993) 529-530.

[12] Warren B.E., X-ray diffraction (Addison-Wesley, London, 1986)

[13] Johnson P.A.V., Wright A.C. and Sinclair R.N.. J. Non-Cryst. Sol. 58 (1983) 109-130.

[14] Poulsen H.F., v. Zimmermann M., Schneider J.R., Schleger P., Hadfield R., Casalta H., Andersen N.H., Ruixing Liang, Dosanjh P. and Hardy N.W., HASYLAB Annual Report (1993) 619-620.

[15] Messoloras S., Schneider J.R., Stewart R.J. and Zulehner W., Semiconductor Sci. Technol.4 (1989) 340-344.

[16] Larson B.C. and Schmatz W., phys. stat. sol. (b) 99 (1980) 267-275.

[17] Bouchard R., Schmidt T., Cornell K., Rütt U., Schneider J.R. and Zulehner W., HASYLAB Annual Report (1993) 615-616.

[18] Lippert M., Brückel T., Köhler T., Schneider J.R. and Jauch W., HASYLAB Annual Report (1993) $621-622$.

[19] Andrews S.R., J. Phys. C19 (1986) 3721-3742.

[20] Shapiro, S.M., Axe J.D., Shirane G. and Riste T., Phys. Rev. B 6 (1972) 4332-4341.

[21] HASYLAB Annual Report (1993) 140-153. 